\title{
Percepção subjetiva de saúde de idosos residentes em instituições de longa permanência
}

\author{
Camila Tomicki*, Ezequiel Vitório Lini ${ }^{\star *}$, Nayam Rafaela de Freitas Picoli***, \\ Luana Cecchin ${ }^{\star \star \star *}$, Marilene Rodrigues Portella ${ }^{\star \star \star \star \star}$
}

\section{Resumo}

A população idosa cresce vertiginosamente e a demanda por serviços especializados para esse público também aumenta, o que torna importante o conhecimento acerca de como esse segmento percebe sua saúde. O objetivo deste estudo foi avaliar a percepção subjetiva da saúde de idosos institucionalizados. Utilizou-se um questionário desenvolvido pelos pesquisadores, com questões objetivas de única escolha, referentes às condições sociodemográficas, clínicas e de autopercepção de saúde. Trata-se de um estudo transversal realizado em instituições de longa permanência para idosos localizadas em um município da região norte do Rio Grande do Sul, Brasil. Realizou-se análise descritiva e inferencial. Na associação entre as variáveis, utilizaram-se os testes Qui-Quadrado de Pearson ou Teste T de Student, em um nível de significância de 5\%. Participaram do estudo trinta pessoas com idades $\geq 60$ anos; $63,3 \%$ do sexo feminino e média de idade de 76,2 anos. Em relação à percepção subjetiva da saúde, 53,3\% consideraram sua saúde como muito boa/boa. Variáveis como idade avançada, presença de comorbidades, polifarmácia e histórico de quedas não demonstraram associação com a autopercepção de saúde. Apesar da elevada presença de doenças e suas complicações, não foi possível afirmar que esses fatores afetaram a percepção subjetiva de saúde dos idosos.

Palavras-chave: Instituição de longa permanência para idosos. Nível de saúde. Saúde do idoso.

\section{Introdução}

A questão do envelhecimento e da longevidade é tema de diversos debates e estudos na atualidade, pois é possível observar um acelerado processo de en-

* Doutoranda do Programa de Pós-Graduação em Educação Física da Universidade Federal de Santa Catarina - UFSC, Florianópolis, SC. Endereço para correspondência: Servidão Euzebia Vidal, 50, Bairro Pantanal, CEP 88040367, Florianópolis, SC. E-mail: camitomicki@gmail.com

** Mestre em Envelhecimento Humano pela Universidade de Passo Fundo, Passo Fundo, RS. E-mail: ezequielfisio@yahoo.com.br

*** Residente em Enfermagem com Ênfase na Saúde do Idoso no Hospital São Vicente de Paulo. Passo Fundo, RS. E-mail: nayrfp@hotmail.com

***** Fisioterapeuta formada pela Universidade de Passo Fundo, Passo Fundo, RS. E-mail: lucecchin@hotmail. com

****** Coordenadora e docente do Programa de Pós-Graduação em Envelhecimento Humano da Universidade de Passo Fundo, Passo Fundo, RS. E-mail: portella@upf.br

$\rightarrow$ http://dx.doi.org/10.5335/rbceh.v13i2.5937 
velhecimento no Brasil e no mundo. $\mathrm{O}$ envelhecimento demográfico é resultado do declínio da fecundidade, da diminuição da taxa de natalidade e do aumento da longevidade, o que não pode ser comparado à qualidade de vida, pois o idoso pode ser longevo, no entanto, apresentar diversas comorbidades que interferem no seu bem-estar (INSTITUTO NACIONAL DE ESTATÍSTICA, 2008).

Devido ao aumento da longevidade, a institucionalização surge como suporte para as famílias que necessitam cuidar do idoso. As instituições disponibilizam um quadro diversificado de profissionais, com o intuito de atender às necessidades do idoso e da família na sua integralidade, pois os motivos que levam os familiares e/ou responsáveis a buscar uma instituição de longa permanência para idosos (Ilpi) são variados, dentre eles: fragilidade decorrente de alguma patologia ou comorbidade, divergências familiares, perda de companheiros, de filhos, entre outros fatores (HERÉDIA, 2012).

A autopercepção do idoso é construída em um contexto múltiplo, tem implicações desde suas vivências culturais, passando por alterações próprias do envelhecimento, sendo influenciada, também, pelo ambiente no qual o indivíduo está inserido. É necessário apontar que a percepção subjetiva negativa da saúde pode ser relacionada a um considerável risco de declínio do idoso, tanto físico quanto mental. Em contrapartida, a autopercepção positiva pode vir como um diagnóstico, apontando a dificuldade em ter senso crítico.
Em relação ao exposto, é importante considerar a percepção subjetiva do estado de saúde do idoso, para então poder criar uma série de ações, visando a melhorias do atendimento, da prevenção e de promoção de saúde e, principalmente, proporcionar satisfação ao indivíduo cuidado. De modo que seja possível ter uma visão mais ampla e efetiva, influenciando positivamente no bem-estar e na qualidade de vida dos longevos, inclusive podendo auxiliar na construção de políticas públicas de saúde voltadas à saúde do idoso (CARVALHO et al., 2012).

Diante desse contexto, este estudo teve por objetivo avaliar a percepção subjetiva da saúde de idosos residentes em Ilpis.

\section{Metodologia}

Trata-se de um estudo transversal realizado em duas Ilpis localizadas em um município da região norte do Rio Grande do Sul, Brasil. O município estudado tem uma população estimada de 195.620 habitantes (INSTITUTO BRASILEIRO DE GEOGRAFIA E ESTATÍSTICA, 2014), e conta com 21 Ilpis, todas privadas, duas, porém, são filantrópicas, totalizando 471 idosos institucionalizados. Nas Ilpis filantrópicas, objeto deste estudo, residem 112 idosos, 39 do sexo masculino e 73 do sexo feminino.

Neste estudo, foram incluídos indivíduos de ambos os sexos, com idade igual ou superior a 60 anos, efetivamente residindo nas instituições e que concordaram em participar do estudo. Dessa forma, a amostra foi composta por trinta idosos. 
A coleta de dados foi realizada por meio de uma entrevista individual, com auxílio de um instrumento desenvolvido pelos pesquisadores com as variáveis: idade, sexo, tempo de institucionalização, estado civil, escolaridade, ocupação anterior bem como dados clínicos de interesse, como doenças, uso de medicamentos, polifarmácia, ocorrência de quedas e fraturas no último ano. Todas as informações foram checadas no prontuário dos residentes e as dúvidas foram esclarecidas com o profissional de enfermagem responsável pelo serviço nas respectivas instituições.

A percepção subjetiva de saúde foi obtida por meio do autorrelato dos participantes sobre como cada um considerava sua própria saúde. A partir da pergunta: $\mathrm{O}(\mathrm{a})$ senhor(a) diria que sua saúde é muito boa, boa, regular, ruim ou muito ruim?, o entrevistador marcava apenas uma das cinco opções de resposta. Após a coleta de dados, percebeu-se que as respostas poderiam ser transformadas em dicotômicas, assim, muito boa e boa formaram um grupo, enquanto regular, ruim e muito ruim formaram outro.

Para a estruturação do banco de dados, utilizou-se o programa Microsoft Excel 2010 e, para as análises dos dados, o software estatístico Statistical Package for Social Science for Windows (SPSS), versão 22.0.

As variáveis numéricas foram expressas como média e desvio padrão ou mediana (percentil ${ }_{25}$ - percentil ${ }_{75}$ ), conforme apresentaram distribuição normal ou não normal. As variáveis categóricas foram expressas como frequência abso- luta e relativa. Consideraram-se como estatisticamente significantes valores de probabilidade $<0,05$.

A pesquisa foi aprovada pelo Comitê de Ética em Pesquisa com Seres Humanos da Universidade de Passo Fundo, sob o protocolo $\mathrm{n}^{\circ} 572.113$, atendendo às diretrizes da Resolução no 466/2012 do Conselho Nacional de Saúde. Todos os participantes receberam informações detalhadas sobre o protocolo de pesquisa e assinaram o termo de consentimento livre e esclarecido.

\section{Resultados}

Participaram do estudo trinta idosos com idade média de 76,2 anos ( $\pm 7,9), 19$ $(63,3 \%)$ do sexo feminino. As características sociodemográficas estão dispostas na Tabela 1. Conforme se observa, houve uma predominância feminina e a maioria dos participantes tinha entre $70 \mathrm{e}$ 79 anos de idade. A maior proporção foi de pessoas viúvas (50\%). Em relação à escolaridade, prevaleceu o ensino fundamental (46,7\%). Como ocupação anterior, houve predominância de atividades braçais, ou seja, atividades que exigiam esforço físico (63,3\%). A média de tempo de institucionalização foi de 28,5 meses, concentrando-se entre 25 e 60 meses (46,7\%), seguida por menos de 24 meses de institucionalização (40\%). 
Tabela 1 - Características sociodemográficas dos idosos residentes em Ilpis, Brasil, $2014(\mathrm{n}=30)$

\begin{tabular}{|c|c|c|}
\hline Variáveis & $\mathrm{n}=30$ & $\%$ \\
\hline Idade (anos) ${ }^{\star}$ & $76,2 \pm 7,9$ & - \\
\hline $\begin{array}{l}\text { Tempo de institucionalização } \\
\text { (meses) }^{\star *}\end{array}$ & $28,5 \pm 52,1$ & - \\
\hline \multicolumn{3}{|l|}{ Sexo } \\
\hline Feminino & 19 & 63,3 \\
\hline Masculino & 11 & 36,7 \\
\hline \multicolumn{3}{|l|}{ Estado civil } \\
\hline Solteiro & 9 & 30,0 \\
\hline Viúvo & 15 & 50,0 \\
\hline Divorciado & 6 & 20,0 \\
\hline \multicolumn{3}{|l|}{ Escolaridade } \\
\hline Analfabeto & 10 & 33,3 \\
\hline Ensino fundamental & 14 & 46,7 \\
\hline Ensino médio & 6 & 20,0 \\
\hline \multicolumn{3}{|l|}{ Ocupação anterior } \\
\hline Atividade braçal & 19 & 63,3 \\
\hline Atividade não braçal & 11 & 36,7 \\
\hline
\end{tabular}

A Tabela 2 resume as características clínicas dos idosos pesquisados quanto à presença de doenças, ao uso de medicamentos, à polifarmácia, a quedas e fraturas. De maneira geral, todos os participantes apresentaram, pelo menos, uma doença crônica. Quanto ao número de medicamentos, houve uma quantidade elevada de medicamentos consumidos por dia (5,8 em média), podendo ser observada a presença da polifarmácia, caracterizada como sendo o uso de cinco ou mais medicamentos de forma concomitante $(76,7 \%)$. Ao verificar o histórico de quedas, foi possível observar uma frequência de 43,3\% de quedas nos últimos doze meses, porém, houve um número pequeno de fraturas (10\%) por quedas, sendo a maioria de colo femoral.

Tabela 2 - Características clínicas dos idosos residentes em Ilpis, Brasil, 2014 $(\mathrm{n}=30)$

\begin{tabular}{l|r|r}
\hline \multicolumn{1}{c|}{ Variáveis } & $\mathrm{n}=30$ & $\%$ \\
\hline Comorbidades & 18 & 60,0 \\
Neurológicas & 20 & 66,7 \\
Cardiovasculares & 4 & 13,3 \\
Psiquiátricas & 3 & 10,0 \\
Ortopédicas & $5,8 \pm 3,1$ & - \\
N ${ }^{\circ}$ de medicamentos & 23 & 76,7 \\
Polifarmácia & 13 & 43,3 \\
Quedas (último ano) & 3 & 10,0 \\
\hline Fraturas (último ano) & & \\
\hline
\end{tabular}

Fonte: primária.

Nota: "Valores que expressam média e desvio padrão ( \pm ).

Em relação à percepção subjetiva da saúde, 53,3\% dos participantes relataram considerar sua saúde como muito boa/boa. Os dados podem ser observados na Tabela 3. 
Tabela 3 - Distribuição dos idosos quanto às variáveis sociodemográficas e clínicas e à percepção subjetiva de saúde, Brasil, $2014(\mathrm{n}=30)$

\begin{tabular}{|c|c|c|c|c|c|}
\hline \multirow{3}{*}{ Variáveis* } & \multicolumn{4}{|c|}{ Percepção subjetiva de saúde } & \multirow[b]{3}{*}{$p$} \\
\hline & \multicolumn{2}{|c|}{ Muito boa/boa } & \multicolumn{2}{|c|}{ Regular/ruim } & \\
\hline & $\mathrm{n}$ & $\%$ & $\mathrm{n}$ & $\%$ & \\
\hline \multicolumn{6}{|l|}{ Sexo } \\
\hline Masculino & 6 & 37,5 & 5 & 35,7 & 0,919 \\
\hline Feminino & 10 & 62,5 & 9 & 64,3 & \\
\hline \multicolumn{6}{|l|}{ Faixa etária } \\
\hline $60-69$ anos & 2 & 12,5 & 4 & 28,6 & 0,183 \\
\hline 70-79 anos & 7 & 43,8 & 8 & 57,1 & \\
\hline$\geq 80$ anos & 7 & 43,8 & 2 & 14,3 & \\
\hline \multicolumn{6}{|l|}{ Tempo de institucionalização } \\
\hline$<24$ meses & 7 & 43,8 & 5 & 35,7 & 0,904 \\
\hline 25 e 60 meses & 7 & 43,8 & 7 & 50,0 & \\
\hline$\geq 61$ meses & 2 & 12,5 & 2 & 14,3 & \\
\hline \multicolumn{6}{|l|}{ Estado civil } \\
\hline Solteiro & 4 & 25,0 & 5 & 35,7 & 0,700 \\
\hline Viúvo & 8 & 50,0 & 7 & 50,0 & \\
\hline Divorciado & 4 & 25,0 & 2 & 14,3 & \\
\hline \multicolumn{6}{|l|}{ Escolaridade } \\
\hline Analfabeto & 6 & 37,5 & 4 & 28,6 & 0,542 \\
\hline Ensino fundamental & 6 & 37,5 & 8 & 57,1 & \\
\hline Ensino médio completo & 4 & 25,0 & 2 & 14,3 & \\
\hline \multicolumn{6}{|l|}{ Doenças } \\
\hline Neurológicas & 8 & 29,6 & 10 & 55,6 & 0,232 \\
\hline Cardiovasculares & 13 & 48,2 & 7 & 38,9 & 0,070 \\
\hline Psiquiátricas & 4 & 14,8 & 0 & 0,0 & 0,044 \\
\hline Ortopédicas & 2 & 7,4 & 1 & 5,5 & 0,626 \\
\hline \multicolumn{6}{|l|}{ Quedas (último ano) } \\
\hline Sim & 8 & 50,0 & 5 & 35,7 & 0,431 \\
\hline Não & 8 & 50,0 & 9 & 64,3 & \\
\hline \multicolumn{6}{|l|}{ Fraturas (último ano) } \\
\hline Sim & 2 & 12,5 & 1 & 7,1 & 0,626 \\
\hline Não & 14 & 87,5 & 13 & 92,9 & \\
\hline \multicolumn{6}{|l|}{ Polifarmácia } \\
\hline Sim & 12 & 75,0 & 11 & 78,6 & 0,818 \\
\hline Não & 4 & 25,0 & 3 & 21,4 & \\
\hline
\end{tabular}

Fonte: primária.

Nota: "Utilizou-se o teste T de Student para as variáveis numéricas e o teste Qui-Quadrado de Pearson para as variáveis categóricas. 
A autopercepção de saúde não demonstrou associação significante quanto ao sexo $(p=0,919)$. Entre os que referiram à própria saúde como regular/ruim, $57,1 \%$ pertenciam à faixa etária entre 70 e 79 anos. Na definição de saúde muito boa/boa, $43,8 \%$ tinham entre 70 e 79 anos e $43,8 \%$ tinham 80 ou mais. Entre os que avaliaram a saúde como regular/ ruim, a maioria $(57,1 \%)$ tinha como nível de escolaridade o ensino fundamental.

Não houve diferença estatisticamente significante na percepção subjetiva de saúde autorreferida quando associada à situação conjugal, assim como não houve associação estatisticamente significante entre o tempo de institucionalização e a percepção subjetiva de saúde dos idosos, sendo que $50 \%$ dos que relataram saúde regular/ruim tinham entre 25 a 60 meses de residência institucional.

Dos que caracterizaram a própria saúde como regular/ruim, 35,7\% sofreram quedas no último ano. Apenas três idosos sofreram quedas que resultaram em fraturas, sem que houvesse correlação estatisticamente significante com a percepção subjetiva de saúde.

Na percepção subjetiva sobre a saúde, $78,6 \%$ dos que referiram ter saúde regular/ruim faziam uso de polifarmácia e 75\% estavam entre aqueles com saúde muito boa/boa. A percepção subjetiva de saúde mostrou, inicialmente, associação significante com a variável doenças psiquiátricas, porém, uma das células apresentou valor igual a zero, o que invalida essa associação. Nas outras doenças ou complicações, não houve associação estatisticamente significante.

\section{Discussão}

É possível observar, na análise descritiva da amostra, que os idosos institucionalizados apresentam, em média, 76,2 anos e a maioria é do sexo feminino $(63,3 \%)$, dado encontrado também em outros estudos, reforçando a feminização da velhice (VAZ; GASPAR, 2011 apud SOARES; COELHO; CARVALHO, 2012).

O tempo de institucionalização teve uma mediana de 28,5 meses, achado que vai ao encontro de outras pesquisas (BARROS; SOUZA; UCHÔA, 2012 apud UCHIDA; BORGES, 2013). Observou-se que todos os participantes apresentaram, pelo menos, uma doença crônica. Conforme Lima e Cezario (2014), a presença de doenças neurológicas, cardiovasculares, psiquiátricas e ortopédicas contribui diretamente para o declínio da capacidade física.

O elevado uso de medicamentos e a presença de polifarmácia foram observados no presente estudo, corroborando com outros estudos realizados com idosos institucionalizados (FOCHAT et al., 2012; RIBEIRO et al., 2013). Em virtude da presença de múltiplas comorbidades e em função da condição crônica há número crescente de idosos que usam medicamentos de modo continuado (HAMRA; RIBEIRO; MIGUEL, 2007), o que requer atenção especial dos profissionais de saúde, pois muitos deles podem ser potencialmente inapropriados para idosos (RIBAS; OLIVEIRA, 2014).

A polifarmácia, definida por Secoli (2010) como o uso de cinco medicamentos ou mais, constitui-se um importante 
problema no atendimento ao idoso, devendo ser evitada sempre que possível, visto que idosos institucionalizados têm risco aumentado para polifarmácia por apresentarem muitas doenças limitantes, fragilidade e baixa funcionalidade (LUCCHETTI et al., 2010).

Em relação às quedas, dos idosos participantes, $13(43,3 \%)$ caíram há menos de um ano. As quedas e suas consequências estão presentes em todas as épocas da vida, no entanto, são especialmente denotadas como um problema de saúde quando na idade mais avançada, haja vista a possibilidade de lesões graves e de impactos psicológicos resultantes desses eventos (COSTA et al., 2011).

A autopercepção de saúde dos idosos é um indicador utilizado com uma frequência considerável, os pesquisadores utilizam esse método por ele ser de baixo custo, além disso, de acordo com diversos estudos, prediz a mortalidade e o declínio funcional dos idosos (BORGES et al., 2014).

É possível constatar, pela literatura, a influência do nível de escolaridade na percepção de saúde dos idosos. Estudos apontam que, em grande parte dos casos, os idosos com maior nível de instrução têm uma percepção positiva quanto à própria saúde, e os idosos com baixa escolaridade tendem a perceber negativamente sua condição de saúde. Tal constatação pode decorrer das oportunidades sociais que surgem ao longo da vida das pessoas com maior grau de instrução, bem como do acesso à informação e da oportunidade de usufruir de mais lazer e melhores condições de vida (BORIM; BARROS; NERI, 2012).
Os sintomas depressivos e a negatividade, assim como a insatisfação com a própria saúde, podem ser resultado de inatividade física, pois, devido ao processo de envelhecimento, geralmente, os idosos reduzem significativamente suas atividades habituais. Desse modo, o sedentarismo contribui para o surgimento de comorbidades e de diversas alterações fisiológicas que comprometem a saúde e o bem-estar do idoso (DOMINGUES; NERI, 2012).

Devido ao envelhecimento, é normal que haja o declínio de aptidões, tais como força, agilidade, flexibilidade e coordenação, o que dificulta a realização das atividades cotidianas e, mais ainda, a realização de atividades físicas que exijam maior desenvoltura. Por conseguinte, é importante que seja elaborado um plano de atividades físicas para ser realizado nas Ilpis, de acordo com as necessidades do grupo, tendo como objetivo maior motivá-los a ser mais ativos e menos sedentários (GONÇALVES et al., 2010).

A necessidade do uso de medicamentos também tem importante influência na percepção subjetiva de saúde do idoso, pois, de acordo com a literatura, o uso contínuo de dois ou mais medicamentos leva o idoso a ter uma percepção negativa de seu estado de saúde. Saber que necessita de variados medicamentos preocupa mais o idoso do que as limitações propriamente ditas resultantes da patologia (SILVA et al., 2012).

Podemos associar os achados do estudo de Tahan e Carvalho (2010) aos resultados do presente estudo, que mostra, de certo modo, a satisfação de 16 (53,3\%) 
idosos institucionalizados com a própria saúde, pois, apesar de apresentarem no mínimo uma doença crônica, afirmam ter boa saúde.

A taxa de autopercepção de saúde muito boa e boa deste estudo (53,3\%) está em desacordo com outros estudos. Na pesquisa de Oliveira e Mattos (2012), a autopercepção de saúde como muito boa e boa foi de $32,8 \%$ dos idosos institucionalizados, enquanto que os achados de Alencar et al. (2012) apresentaram taxas mais elevadas (72,3\%). A subjetividade dessa avaliação pode explicar a divergência que existe entre os estudos.

É possível observar que os idosos relacionam boa saúde com autonomia e independência, e não apenas com ausência de doenças. Além disso, é necessário salientar que a comparação com outros indivíduos é um fator importante na percepção de saúde, pois a comparação torna mais leve o enfrentamento de situações negativas quando o sujeito se compara a alguém em situação pior que a sua (SILVA et al., 2012).

Uma das limitações do presente estudo consiste no tamanho reduzido da amostra, o que não permite inferir generalizações, todavia, os dados alertam os profissionais envolvidos com as Ilpis sobre a percepção de saúde dos idosos e as principais condições que influenciam na avaliação da saúde. Sugere-se que se realizem novos estudos que incluam uma amostra representativa e a comparação entre a percepção subjetiva de idosos residentes em Ilpis a fim de visualizar possíveis diferenças e a aplicação de questionários relacionados à percepção de saúde a fim de se obter uma melhor avaliação da variável estudada.

\section{Conclusão}

Dentre os resultados deste estudo, vale ressaltar a presença de doenças e comorbidades, principalmente cardiovasculares e neurológicas. A taxa de quedas no último ano mostrou-se elevada, assim como a prática de polifarmácia. No entanto, esses fatores não interferiram na autopercepção de saúde dos idosos.

A autopercepção de saúde mostrou-se divergente de outros estudos, no entanto, o questionamento de como o idoso institucionalizado percebe sua saúde é, de fato, muito subjetiva, com influência de diversos fatores, tanto físicos quanto psicossociais.

Ações promovidas por diferentes profissionais, direcionadas à prevenção, à reabilitação, à manutenção e/ou melhora da capacidade funcional, ao estímulo ao lazer e à recreação, podem repercutir em maior qualidade de vida e bem-estar físico e mental, o que influencia direta e positivamente na percepção de saúde dos idosos institucionalizados.

\section{Health subjective perception of elderly in residents in long-term institutions}

\section{Abstract}

The elderly population grows dramatically and the demand for specialized services to this group increases, which makes it important to know about how this segment realize their health. The objective was to

RBCEH, Passo Fundo, v. 13, n. 2, p. 219-228, maio/ago. 2016 
evaluate the subjective perception of health of institutionalized elderly. We used a questionnaire developed by researchers with objective questions of only choice, regarding sociodemographic, clinical conditions and self perception health. It is a cross-sectional study conducted in ILPIs a municipality located in the northern region of Rio Grande do Sul, Brazil. A descriptive and inferential analysis. The association between the variables we used the chi-square test of Pearson or Student's t test at $5 \%$ significance level. The study included 30 people aged $\geq 60$ years, $63.3 \%$ female, mean age 76,2 years. Regarding the subjective perception of health, $53.3 \%$ considered their health as very/good. Variables such as age, comorbidities, polypharmacy, history of falls showed no association with self-perceived health. Despite the high presence of diseases and their complications, it was not possible to say that these factors affect the subjective perception of health of the elderly.

Keywords: Health of the elderly. Health status. Homes for the aged.

\section{Agradecimento}

\section{À Fundação de Amparo à Pesquisa do Estado do Rio Grande do Sul.}

\section{Referências}

ALENCAR, M. A. et al. Perfil dos idosos residentes em uma instituição de longa permanência. Revista Brasileira de Geriatria e Gerontologia, Rio de janeiro, v. 15, n. 4, p. 785-796, 2012.

BORGES, A. M. et al. Autopercepção de saúde em idosos residentes em um município do interior do Rio Grande do Sul. Revista Brasileira de Geriatria e Gerontologia, Rio de Janeiro, v. 17, n. 1, p. 79-86, 2014.
BORIM, F. S.; BARROS, M. B.; NERI, A. L. Autoavaliação da saúde em idosos: pesquisa de base populacional no Município de Campinas, São Paulo. Caderno de Saúde Pública, Rio de Janeiro, v. 28, n. 4, p. 769-780, abr. 2012.

CARVALHO, F. F. et al. Análise da percepção do estado de saúde dos idosos da região metropolitana de Belo Horizonte. Revista Brasileira de Geriatria e Gerontologia, v. 15, n. 2, p. 285-293, 2012.

COSTA, A. G. S. et al. Acidentes por quedas em um grupo específico de idosos. Revista Eletrônica de Enfermagem, Goiânia, v. 13, n. 3, p. 395-404, 2011.

DOMINGUES, P. C.; NERI, A. L. Atividade física habitual, sintomas depressivos e doenças auto-relatadas em idosos da comunidade. Revista Brasileira de Atividade Física \& Saúde, Pelotas, v. 14, n. 3, p. 164-173, 2009.

FOCHAT, R. C. et al. Perfil de utilização de medicamentos por idosos frágeis institucionalizados na Zona da Mata Mineira, Brasil. Revista de Ciências Farmacêuticas Básica e Aplicada, Araraquara, v. 33, n. 3, p. 447-454, 2012.

GONÇALVES, L. H. T. et al. O idoso institucionalizado: avaliação da capacidade funcional e aptidão física. Caderno de Saúde Pública, Rio de Janeiro, v. 26, n. 9, p. 17381746, set. 2010. Disponível em: <http://www. scielo.br/scielo.php?script=sci_arttext\&pid= S0102311X2010000900007\&lng=en\&nrm=i so>. Acesso em: 26 jun. 2015.

HAMRA, A.; RIBEIRO, M. B.; MIGUEL, O. F. Correlação entre fratura por queda em idosos e uso prévio de medicamentos. Acta Ortopédica Brasileira, v. 15, n. 3, p. 143-145, 2007.

HERÉDIA, V. B. M. Abandono na velhice: idosos vivem dor silenciosa ao serem deixados de lado. 2012. Disponível em: <http://idmed. com.br/saude-do-idoso/abandono-na-velhice-idosos-vivem-dor-silenciosa-ao-serem-deixados-de-lado.html>. Acesso em: 16 dez. 2014. 
INSTITUTO BRASILEIRO DE GEOGRAFIA E ESTATÍSTICA. Estatística. População. Diretoria de Pesquisas, Coordenação de População e Indicadores Sociais. 2014. Disponível em: <http://www.ibge.gov.br/home/ estatistica/populacao/estimativa2014/estimativa_tcu.shtm>. Acesso em: 12 jun. 2015.

INSTITUTO NACIONAL DE ESTATÍSTICA. Estatísticas demográficas. Lisboa: Instituto Nacional de Estatística, 2008.

LIMA, D. A.; CEZARIO, V. O. B. Quedas em idosos e comorbidades clínicas. Revista HUPE, Rio de Janeiro, v. 3, n. 2, p. 30-37, 2014.

LUCCHETTI, G. et al. Fatores associados à polifarmácia em idosos institucionalizados. Resultados da pesquisa. Revista Brasileira de Geriatria e Gerontologia, Rio de Janeiro, v. 13, n. 1, p. 51-58, 2010.

OLIVEIRA, P. H.; MATTOS, I. E. Prevalência e fatores associados à incapacidade funcional em idosos institucionalizados no município de Cuiabá, Estado de Mato Grosso, Brasil, 2009-2010. Epidemiologia e Serviços de Saúde, Brasília, v. 21, n. 3, p. 395-406, 2012.

RIBAS, C.; OLIVEIRA, K. R. Perfil dos medicamentos prescritos para idosos em uma Unidade Básica de Saúde do município de Ijuí-RS. Revista Brasileira de Geriatria e Gerontologia, Rio de Janeiro, v. 17, n. 1, p. 99-114, 2014.

RIBEIRO, N. P. et al. Polifarmácia utilizada por idosos residentes em instituições de longa permanência do município de Viamão/RS. Revista Ciência e Movimento, v. 15, n. 30, p. 65-74, 2013.

SECOLI, S. R. Polifarmácia: interações e reações adversas no uso de medicamentos por idosos. Revista Brasileira de Enfermagem, Brasília, v. 63, n. 1, p. 136-140, 2010.

SILVA, R. J. S. et al. Prevalência e fatores associados à percepção negativa da saúde em pessoas idosas no Brasil. Revista Brasileira de Epidemiologia, Sergipe, v. 15, n. 1, p. 49-62, 2012.
TAHAN, J.; CARVALHO, A. C. de. Reflexões de idosos participantes de grupos de promoção de saúde acerca do envelhecimento e da qualidade de vida. Saúde e Sociedade, São Paulo, v. 19, n. 4, 2010. Disponível em: <http://www.scielo.br/scielo.php?script=sci arttext\&pid=S0104-12902010000400014\&ln $\mathrm{g}=\mathrm{en} \& \mathrm{nrm}=\mathrm{iso}>$. Acesso em: 30 jun. 2015.

UCHIDA, J. E. F.; BORGES, S. M. Quedas em idosos institucionalizados. Revista Kairós Gerontologia, v. 16, n. 3, p. 83-94, 2013.

VAZ, S. F.; GASPAR, N. M. Depressão em idosos institucionalizados no distrito de Bragança. Revista Enfermagem, v. 3, n. 4, p. 49-58, 2011. 